\title{
Nucleotide Sequence Comparison of the Mycobacterial dnaJ Gene and PCR-Restriction Fragment Length Polymorphism Analysis for Identification of Mycobacterial Species
}

\author{
SYUN-ICHI TAKEWAKI,${ }^{1} \dagger$ KATSUKO OKUZUMI, ${ }^{1}$ ICHIRO MANABE, ${ }^{2}$ MASAKO TANIMURA, ${ }^{3}$ \\ KIKUKO MIYAMURA, ${ }^{4}$ KEN-ICHI NAKAHARA, ${ }^{1}$ YOSHIO YAZAKI, ${ }^{2}$ \\ AKIYUKI OHKUBO, ${ }^{1}$ AND RYOZO NAGAI ${ }^{1,2 *}$ \\ Department of Laboratory Medicine ${ }^{1}$ and The 3rd Department of Internal Medicine, ${ }^{2}$ University of Tokyo, \\ Bunkyo, Tokyo 113; Department of Child Ecology, National Children's Medical Research Center, ${ }^{3}$ Setagaya, \\ Tokyo 154; and Department of Epidemiology, National Institute of Health, ${ }^{4}$ Shinjuku, Tokyo 162, Japan

\begin{abstract}
We recently reported a genus-specific PCR for the mycobacterial dnaJ gene. In the present study, we have determined the nucleotide sequences of the dnaJ gene from 19 mycobacterial species (Mycobacterium tuberculosis, M. bovis, M. bovis BCG, M. africanum, M. microti, M. marinum, M. kansasii, $M$. gastri, $M$. simiae, M. scrofulaceum, M. szulgai, M. gordonae, M. avium, M. intracellulare, M. xenopi, $M$. fortuitum, $M$. chelonae, $M$. hemophilum, and $M$. paratuberculosis). On the basis of the amplified dnaJ gene nucleotide sequences, we constructed a phylogenetic tree of the mycobacterial species by using the neighbor-joining method and unweighted pairwise grouping method of arithmetic average. We found that the phylogenetic relationship inferred within the slowly growing species was in good agreement with the traditional classification, with three major branches corresponding to Runyon's groups I, II, and III. An exception was M. simiae, which was phylogenetically closer to the cluster including members of Runyon's group III than to that of Runyon's group I. On the other hand, the rapid growers, such as $M$. fortuitum and $M$. chelonae, did not form a coherent line corresponding to Runyon's group IV, indicating that our phylogenetic analysis based on the $d n a J$ gene reflects the phenotypic characteristics such as pigmentation but not the growth rate. Finally, we revealed the species-specific restriction sites within the amplified dnaJ gene to differentiate most of the mycobacterial DNA by a combination of PCR with restriction fragment length polymorphism analysis.
\end{abstract}

Tuberculosis, a major health problem in the developing world, is still recognized as a continuing problem even in developed countries. Nontuberculous mycobacteria, the pathogenic potential of which is weaker than that of $\mathrm{Myco}$ bacterium tuberculosis, can also cause clinically serious infectious diseases (11). Conventional bacteriological determination of the mycobacterial species takes 3 to 8 weeks for culture and another 2 to 4 weeks for final identification by biochemical analyses. DNA-RNA hybridization assay is a new approach for identifying mycobacterial species by genotype, which reduces the time required for identification at the species level. DNA probes specific for rRNA of the $M$. tuberculosis complex, $M$. avium, and $M$. intracellulare have been introduced into clinical bacteriology $(9,16)$. PCR for mycobacterial DNA has also been developed for direct detection and identification of the mycobacterial species in clinical samples $(4-6,19,21,30,32,40)$. We recently reported a genus-specific PCR for the mycobacterial dnaJ gene (35). The dnaJ gene is a stress protein-coding gene, and is highly conserved among bacterial genera $(8,41)$. In this study, we present the nucleotide sequences of the amplified $d n a J$ genes from 19 mycobacterial species and show that the $d n a J$ gene sequences are useful not only for identification of mycobacterial species but also for inferring their phylogenetic relationship.

A fundamental taxonomic division of mycobacteria has

\footnotetext{
* Corresponding author. Mailing address: The 3rd Department of Internal Medicine, University of Tokyo, 7-3-1 Hongo, Bunkyo, Tokyo 113, Japan. Phone: (81)-3-3815-5411, ext. 8272. Fax: (81)-33815-2087.

† Present address: Iatron Laboratories, Inc., Chiyoda, Tokyo 113 , Japan.
}

been based on growth rate and pigmentation. By these criteria, the genus Mycobacterium has been divided into four Runyon groups: group I, the photochromogenic species of slow growers; group II, the scotochromogenic species of slow growers; group III, the nonphotochromogenic species of slow growers; and group IV, the rapid growers $(24,25)$. Further attempts to subdivide the mycobacterial species have been made using immunological techniques (38), comparison of cell wall components (15), and DNA-DNA hybridization $(2,3,10,12)$, but these provide little phylogenetic information. Recently, nucleotide sequence comparison of the 16S rRNA genes was systematically used to infer phylogenetic relationships among the mycobacteria $(20,23,34)$. However, the phylogeny based on the 16S rRNA gene did not reflect Runyon's classification of slowly growing mycobacteria in all cases. To clarify the relationship between the Runyon groups and the phylogeny of mycobacterial species, the nucleotide sequences of various genes must be compared. In particular, nucleotide sequences of a proteincoding gene should provide new insights into phylogenetic relatedness among mycobacteria.

In this study, we present the nucleotide sequences of the dnaJ gene from 19 mycobacterial species and their phylogenetic relationships. We also demonstrate that, when combined with the restriction fragment length polymorphism (RFLP) analysis following amplification, the PCR assay for the dnaJ gene should provide a clinically useful method of detecting and identifying mycobacteria.

\section{MATERIALS AND METHODS}

Bacterial strains. Bacteria examined in this study are listed in Table 1. They were obtained from the National Institute of 
TABLE 1. Mycobacteria examined in this study

\begin{tabular}{|c|c|}
\hline Species $^{a}$ & Strains $^{b}$ \\
\hline \multicolumn{2}{|c|}{ M. africanum ................................................. } \\
\hline M. avium.......... & . NIHJ 1605 \\
\hline M. bovis... & .. NIHJ 1607 \\
\hline M. bovis BCG.. &. KK $12-02$ \\
\hline M. chelonae..... &. NIHJ 1611 \\
\hline M. fortuitum & ..NIHJ 1615 \\
\hline M. gastri..... & ..NIHJ 1616 \\
\hline M. gordonae... & ..NIHJ 1617 \\
\hline M. hemophilum &. KK $49-01$ \\
\hline M. intracellular & .ATCC $13950^{\mathrm{T}}$ \\
\hline M. kansasii... & ..ATCC $12478^{\mathrm{T}}$ \\
\hline M. marinum... & .. NIHJ 1620 \\
\hline M. microti.... & $. . \mathrm{KK} 14-10$ \\
\hline M. paratuberc & ..ATCC $19698^{\mathrm{T}}$ \\
\hline M. scrofulaceun & .. NIHJ 1626 \\
\hline M. simiae ........ & ..NIHJ 1627 \\
\hline M. szulgai .... & ..NCTC 10831 \\
\hline M. tuberculosis. & .. NIHJ 1633 \\
\hline M. xenopi..... & .. NIHJ 1638 \\
\hline
\end{tabular}

${ }^{a}$ In alphabetical order.

${ }^{b}$ Type strains are indicated by $\mathrm{T}$.

Health (Tokyo, Japan), American Type Culture Collection (Rockville, Md.), or National Collection of Type Cultures (London, England). All species were inoculated on 1\% Ogawa egg medium (18) and confirmed by standard biochemical procedures (33).

PCR. We earlier reported the development of PCR primers for detecting the dnaJ gene of mycobacteria (35). Briefly, primer set 1 could successfully amplify the dnaJ gene of 18 mycobacterial species as a 236-bp DNA fragment (5'-GGGT GACGCGGCATGGCCCA-3', 5'-CGGGTTTCGTCGTACT CCTT-3'). To amplify the dnaJ gene of $M$. chelonae, primer set 2 was used for the PCR (5'-AGAGGGGGTGACGCGA CATG-3', 5'-TCGTCGTACTCCTTGCGCTT-3'). All PCR primers were synthesized on a $380 \mathrm{~A}$ DNA synthesizer (Applied Biosystems, Foster City, Calif.) with $\beta$-cyanoethyl phosphoramidite chemistry. All solutions and Taq DNA polymerase were purchased from Perkin-Elmer Cetus (GeneAmp kit; Perkin-Elmer Cetus, Norwalk, Conn.). For the PCR assay, all reactions were performed according to the conventional method with a final volume of $50 \mu$ l containing $100 \mathrm{ng}$ of purified DNA from various bacterial strains, $1 \times$ reaction buffer (GeneAmp), $200 \mu \mathrm{M}$ deoxynucleoside triphosphate mixture, 50 pmol of each primer, and $1 \mathrm{U}$ of Taq polymerase (26). Amplification was carried out by 38 cycles on a programmable thermal cycler (Perkin-Elmer Cetus). Each cycle was performed with heat denaturation at $94^{\circ} \mathrm{C}$ for $30 \mathrm{~s}$, primer annealing at $65^{\circ} \mathrm{C}$ for $1 \mathrm{~min}$, and extension at $72^{\circ} \mathrm{C}$ for $2 \mathrm{~min}$. Extensive care was taken during the experiment to avoid DNA contamination (13).

Nucleotide sequence analysis of the amplified PCR products. The identity of the amplified DNA with the target sequences of the dnaJ gene was confirmed by nucleotide sequencing analysis which was performed by the dideoxy chain termination method (29) following subcloning of the PCR products into M13mp18. Briefly, the amplified DNA fragment was purified by extraction from agarose gel after electrophoresis and treated with T4 DNA polymerase and Klenow fragment (Toyobo, Osaka, Japan) for blunt-ending (28) and then ligated into SmaI-digested M13mp18. We determined the nucleotide sequences by using at least four subclones to eliminate any incorrect sequences caused by misincorporation of nucleotides during PCR.
Phylogenetic analysis. The nucleotide sequences of the amplified dnaJ genes from 19 mycobacteria were used for all pairwise comparisons. They were aligned by using the multisequence alignment algorithm in the program package Clustal V. Phylogenetic relationships were inferred by two kinds of distance matrix methods. One was inferred by the neighbor-joining method (27), and the other tree was constructed by unweighted pairwise grouping method of arithmetic average (UPGMA) (36). To indicate topological errors in the unrooted tree by the neighbor-joining method, we used the bootstrapping technique (7), which involves resampling points from one's own data with replacements, to create a series of bootstrap samples of the same size as the original data. We used 1,000 bootstrap replicates and evaluated the topology of the tree. A phylogenetic relationship based on deduced dnaJ amino acid sequences from 19 mycobacterial dnaJ gene fragments was also inferred by the neighborjoining method.

PCR-RFLP analysis. Five microliters of the amplified PCR products was digested with SmaI, NaeI, HinfI, and FokI according to manufacturers' instructions. After digestion, the reaction mixtures were electrophoresed on $3.5 \%$ Nusieve agarose gel and visualized by UV fluorescence.

\section{RESULTS}

Amplification of dnaJ genes. We already reported primer development of the PCR for the dnaJ genes of mycobacteria (35). The PCR for the $d n a J$ gene with primer set 1 gave rise to a 236-bp fragment in 18 tuberculous and nontuberculous mycobacterial species (Fig. 1); $M$. chelonae could only be successfully amplified to a 225 -bp fragment with primer set 2 . The 44 nonmycobacterial species frequently isolated from the respiratory system were also subjected to amplification but did not show any amplified DNA fragments (data not shown).

Nucleotide and amino acid sequences of $d n a J$ genes from 19 mycobacterial species. Nucleotide sequences of gene segments of the dnaJ gene were determined for 19 mycobacterial species by the dideoxy termination method. The nucleotide and deduced amino acid sequences of the dnaJ genes of those mycobacteria are shown in Fig. 2. Among the tuberculosis complex species, such as $M$. tuberculosis, $M$. bovis, $M$. bovis BCG, $M$. africanum, and $M$. microti, the nucleotide sequences of the amplified $d n a J$ genes were completely identical. There were two characteristic differences in the nucleotide sequences of the amplified dnaJ genes of the $M$. tuberculosis complex and the nontuberculous mycobacterial groups. First, nucleotides from positions 1415 to 1417 (AGG) of the M. tuberculosis dnaJ gene were substituted at the first letter (A to $C$ ) and the third letter ( $G$ to $\mathrm{T}, \mathrm{A}$, or $\mathrm{C}$ ) of the codon without amino acid changes in nontuberculous species. Second, nucleotide position 1442 was substituted from $\mathrm{C}$ to $\mathrm{A}$ in the nontuberculous species, leading to one amino acid change from Gin to Lys ( $Q$ to $\mathrm{K}$ ). These distinctive features make the $M$. tuberculosis complex distinguishable from nontuberculous mycobacteria. Nontuberculous mycobacteria could be further classified into several major groups based on unique substitutions both at the nucleotide and amino acid levels. $M$. kansasii, $M$. marinum, $M$. gastri, and $M$. chelonae showed three unique substitutions at amino acid levels which were not found in other species: at residue 31 , Tyr to $\mathrm{Ala}$ (Y to A); at residues 34 to 37, Ala-Arg-Asp-Leu to Leu-Ala-Glu-Asn (ARDL to LAEN); and at residues 59 to 60 , His-Asn to Lys-Glu or to Lys-Asp (HN to KE or KD). M. scrofulaceum, M. szulgai, 


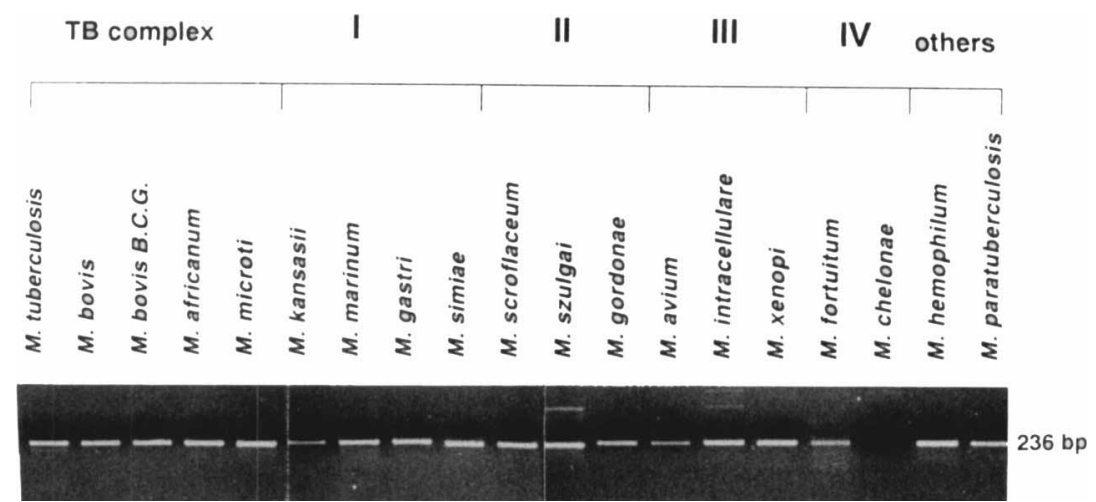

FIG. 1. Amplification of the $M$. tuberculosis complex and other mycobacterial dnaJ genes by PCR with primer set 1 . Eighteen tuberculous and nontuberculous members of the genus Mycobacterium could be amplified as 236-bp DNA fragments. Mycobacterial groups classified by Runyon are indicated at the top of the figure $(24,25)$. The PCR products were analyzed on $3.5 \%$ Nusieve agarose gel by electrophoresis.

and $M$. gordonae, all of which belong to Runyon's group II, showed several unique amino acid substitutions at residue 24 , Glu to Lys ( $\mathrm{E}$ to $\mathrm{K}$ ); residue $28, \operatorname{Arg}$ to $\mathrm{Thr}$ ( $\mathrm{R}$ to $\mathrm{T}$ ); residue 35 , Arg to Ser ( $R$ to $S$ ); residue 41 , Ala to Lys (A to $\mathrm{K}$ ); residues 48 and 49 , Ala-Gly to Gly-Ala (AG to GA); residues 59 and 60 , His-Asn to Tyr-Ser (HN to YS); and residue 65, Pro to Glu (P to E). Nucleotides from positions 1535 to 1543 were found to be deleted in $M$. scrofulaceum, $M$. szulgai, and $M$. gordonae.

Phylogenetic tree of mycobacterial species derived from dnaJ gene nucleotide sequence analysis. Table 2 summarizes the similarities of the nucleotide sequences among the mycobacterial dnaJ genes. As expected, $M$. avium and $M$. intracellulare showed a great similarity (94\%). M. avium and $M$. paratuberculosis were almost identical (99\%). Similarity between $M$. kansasii and $M$. gordonae was the lowest among all of the pairwise comparisons $(62 \%)$.

The phylogenetic relationship of mycobacterial species was inferred by the neighbor-joining method and UPGMA based on the nucleotide differences of the dnaJ gene. The neighbor-joining method gives a topological relationship among the mycobacterial species without taking account of the evolutionary rate. Nineteen mycobacterial species were used for construction of a phylogenetic tree (Fig. 3, top). The tree showed three clusters: the first consisted of the $M$. tuberculosis complex, $M$. avium, $M$. intracellulare, $M$. xenopi, $M$. simiae, $M$. hemophilum, and $M$. paratuberculosis; the second cluster consisted of $M$. kansasii, $M$. gastri, $M$. marinum, and $M$. chelonae; and the third cluster consisted of $M$. scrofulaceum, $M$. gordonae, $M$. szulgai, and $M$. fortuitum. We found that the phylogenetic relationship derived from the dnaJ gene sequence analysis was in good agreement with Runyon's traditional classification in slowly growing mycobacteria, but there was an exception: $M$. simiae (Runyon's group I) showed a closer relationship to $M$. avium and $M$. intracellulare (Runyon's group III) than other members of Runyon's group I, such as $M$. kansasii or $M$. gastri, in our phylogenetic analysis. The rapidly growing mycobacteria, $M$. chelonae and $M$. fortuitum, did not form a coherent line like the slowly growing mycobacteria.

A rooted phylogenetic tree was constructed by using UPGMA based on the similarity values in Table 1 . The tree inferred by UPGMA indicated that mycobacterial species were derived from a common ancestor and then diverged into two branches. The first branch consisted of $M$. kansasii, $M$. marinum, M. gastri, and $M$. chelonae. The second branch containing 15 out of 19 mycobacteria immediately diverged into two branches. As a result, the tree was made up of three major clusters, showing that a topological relationship by UPGMA was similar to that inferred by the neighbor-joining method except for the line consisting of $M$. hemophilum (Fig. 3, bottom).

By the neighbor-joining method (Fig. 3, top), only short nucleotide sequences of the dnaJ gene were aligned. Thus, to evaluate the reliability of the phylogenetic tree inferred by this method, we also used the bootstrap method. The values in the phylogenetic tree represent bootstrap probabilities. The evolutionary relationship consisting of three branches was statistically significant, because the bootstrap probabilities for occurrence of a tree with three branches were not less than 0.95 .

Phylogenetic relationship based on deduced $d n a J$ amino acid sequences. To further test our proposed phylogenetic relationship and its relationship to the conventional classification, we constructed a phylogenetic tree derived from dnaJ amino acid sequence analysis by the neighbor-joining method. As shown in Fig. 4, the topological relationship inferred from the dnaJ amino acid sequence differences was very similar to that based on the dnaJ gene nucleotide sequences.

PCR-RFLP analysis for differentiation of mycobacterial species. The nucleotide sequences of the dnaJ gene which were amplified by PCR revealed several unique endonuclease restriction sites for 19 mycobacterial species (Fig. 5), indicating that the restriction mapping of the PCR products can provide a convenient method to classify the mycobacterial DNA without nucleotide sequencing. For instance, the dnaJ gene of $M$. tuberculosis in the region between sequence positions 1394 and 1629 has one SmaI site and one NaeI site. The $M$. avium dnaJ gene in the same region has a single SmaI site and no NaeI site. From these observations, we felt that the mycobacterial species might be discriminated by RFLP analysis of the amplified dnaJ gene. RFLP by SmaI, NaeI, HinfI, and FokI digestion of the PCR products is shown in Fig. 6. From the PCR-RFLP analysis, 19 species could be discriminated as 10 groups. In particular, clinically important major mycobacteria, such as $M$. tuberculosis, $M$. avium, $M$. intracellulare, and $M$. kansasii, were found to be distinguishable by this method. $M$. kansasii, $M$. marinum, and $M$. gastri, however, have the same restriction sites for SmaI, HinfI, and FokI. M. avium and M. paratuberculosis, which are known as genetically close species (31), have also 
M. tuberculosi. 1414 a AGg GAA TGG GTC GAA AAA GAC TTC TAC CAG GAG CTG GGC GTC TCC TCT GAT GCC AGT CCT GAA GAG ATC M. bovis M. bovis BCG M. africanum M. microti M. kansasi

M. marinum

M. gastri

M. simive

M. scroflaceum

M. szulgai

M. gordonae

M. intracellulare

M. avium

M. xenopi

M. fortuitum

M. chelonae

M. hemophilum

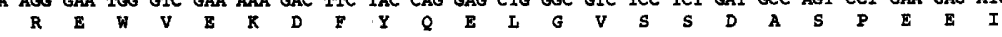

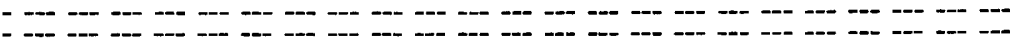

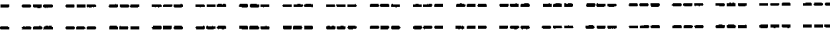

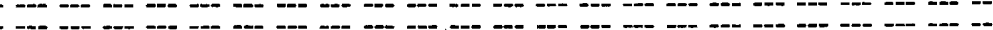

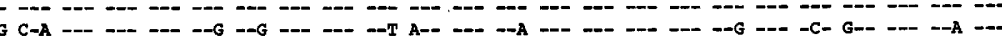

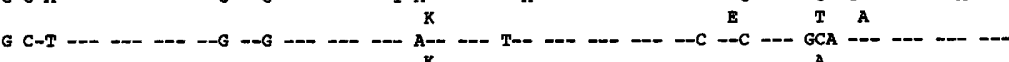
$\mathrm{K} \quad \mathrm{A}$

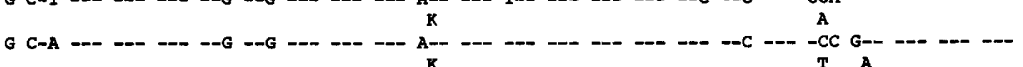

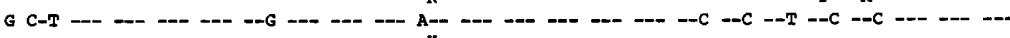

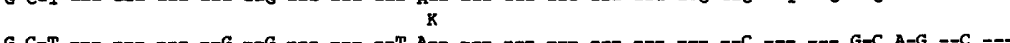

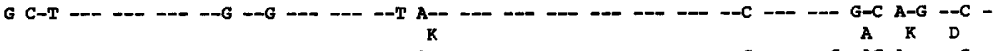

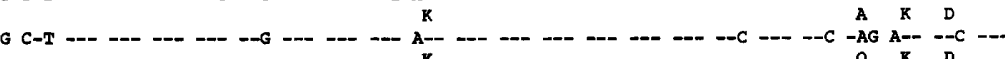
G C-T -.. -.. -.. -.. -.. -.. -. -

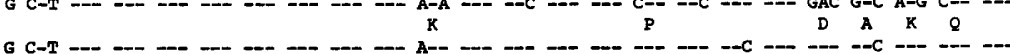
G C-T -- -D - - - - - - -

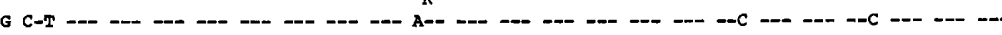

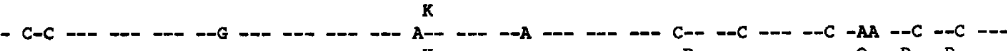

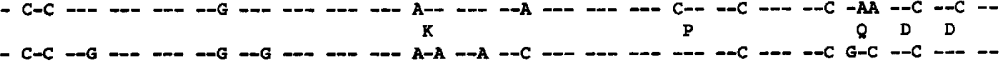
- C-C --G --- --- --G --G --- --. ---

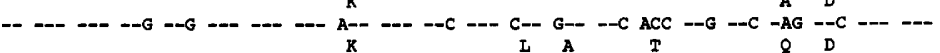

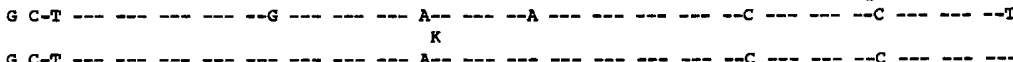

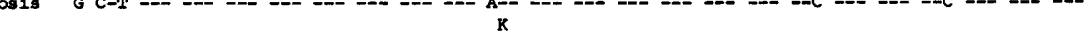

M. tuberculosis 1484 AAA CGT GCC TAT CGg AAG TTG GCG CGC GAC CTG CAT CCG GAC GCG AAC CCG GGC AAC CCG GCC GCC GGC $\begin{array}{llllllllllllllllllllllll}\mathbf{K} & \mathbf{R} & \mathbf{A} & \mathbf{Y} & \mathbf{R} & \mathbf{K} & \mathbf{L} & \mathbf{A} & \mathbf{R} & \mathbf{D} & \mathbf{L} & \mathbf{H} & \mathbf{P} & \mathbf{D} & \mathbf{A} & \mathbf{N} & \mathbf{P} & \mathbf{G} & \mathbf{N} & \mathbf{P} & \mathbf{A} & \mathbf{A} & \mathbf{G}\end{array}$ M. bovi: M. bovis BCG M. africanum M. microti M. kansasii

M. marinum

M. gastri

M. simise

M. scroflaceum

M. Bzulgai

M. gordonae

M. intracellulare

M. avium

M. xenopi

M. fortuitum

M. chelosae

M. hemophilum

M. paratubersulosis

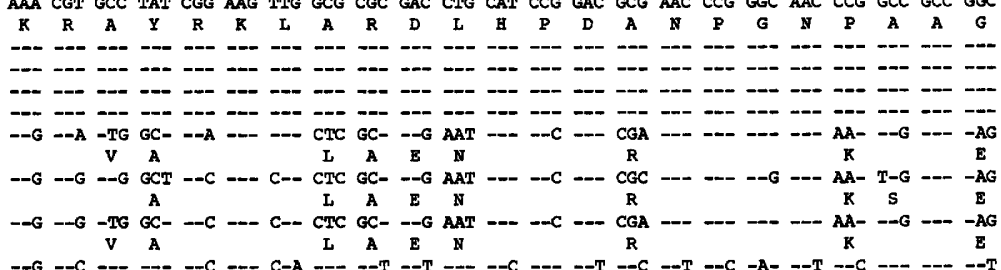

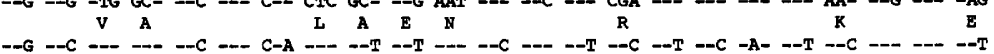

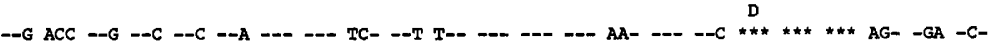

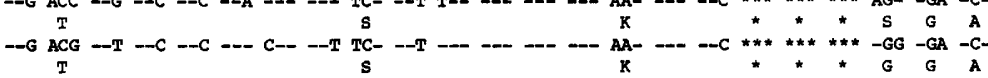

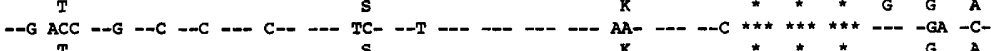

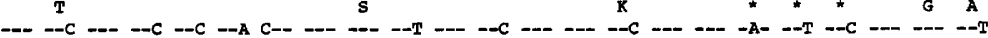
---C --- --C - - -

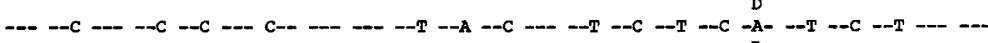
$-C-C$ -

$--G$--C --- --C --C --- C-- --- --- --A --- --C --C --- --C --T -GC AA- G-T --- CG- --T -CA

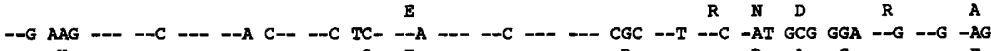

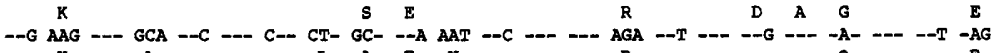

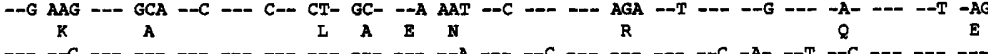

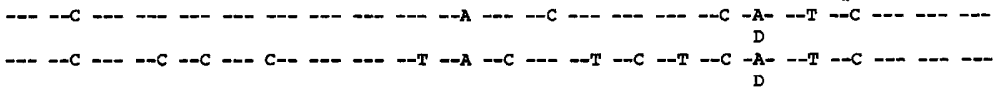

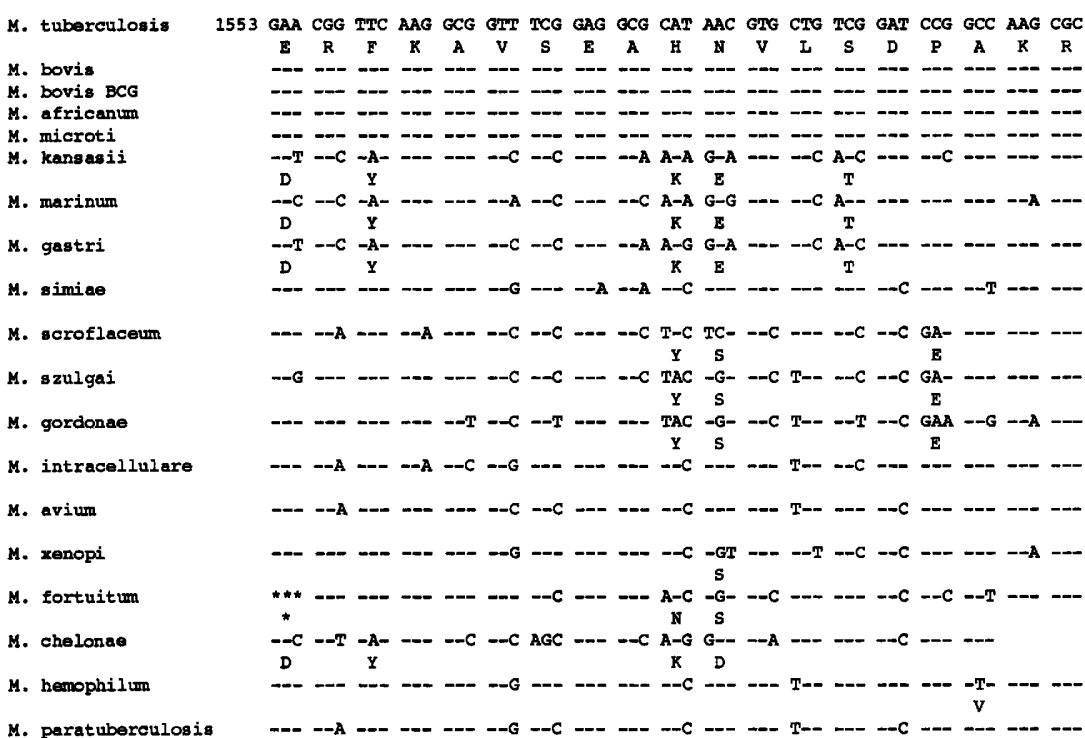

FIG. 2. Nucleotide and amino acid sequences of the $d n a J$ gene segments of 19 mycobacterial species between sequences 1414 and 1609 . The numbers of the nucleotides are given at the beginning of the sequence, according to Lathigra et al. (14), and the deduced amino acid sequence is shown in single-letter code. Nucleotides and amino acids are noted only where different from those of $M$. tuberculosis; where identical, they are shown by dashes. *, deleted nucleotides or amino acids. 
TABLE 2. Pairwise similarity between mycobacterial species ${ }^{a}$

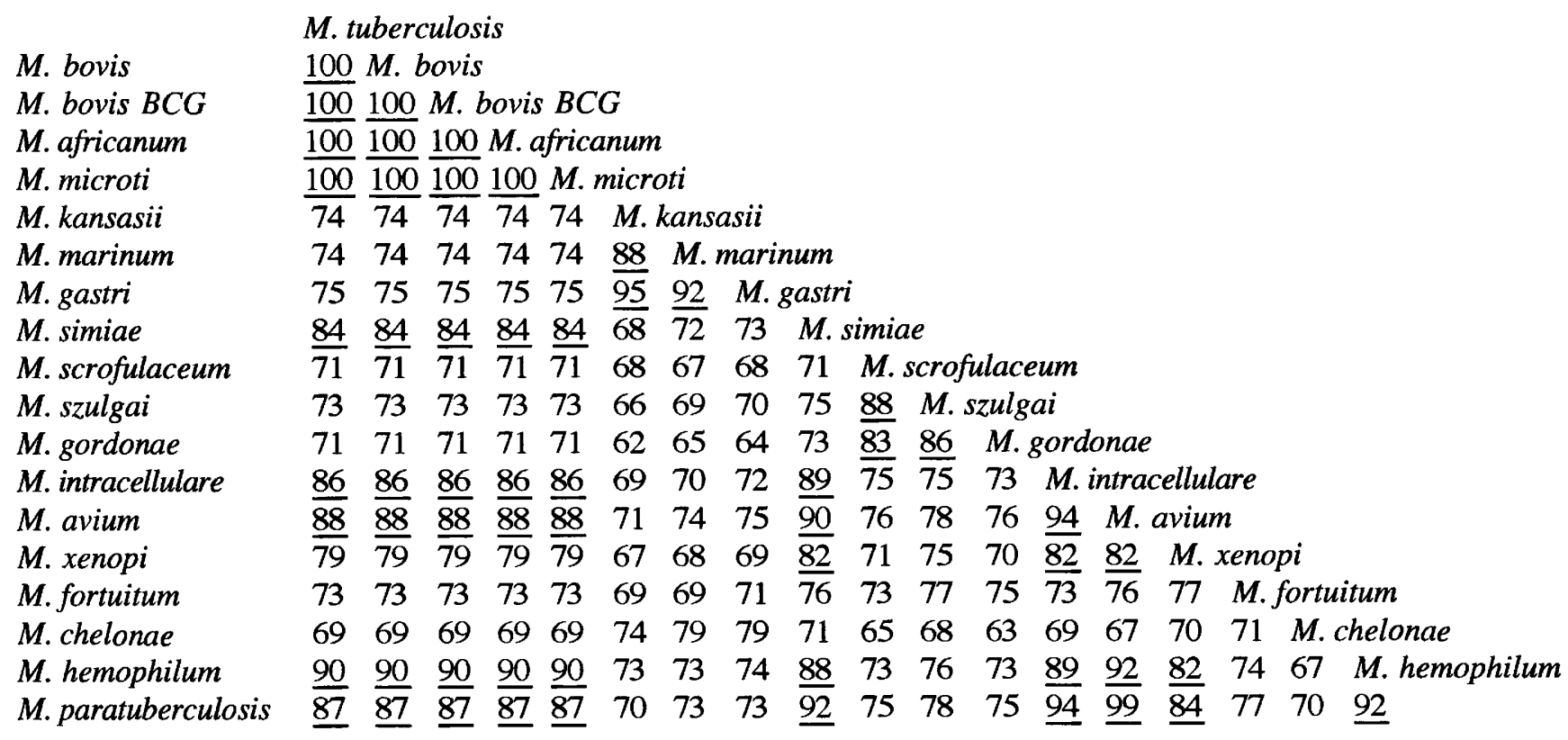

${ }^{a}$ Figures represent the percentage of the nucleotide sequence identity between mycobacterial species within the 196 nucleotides of the dnaJ gene. $M$. chelonae was compared with other species pairwise as to 185 nucleotide sequences. Values larger than $80 \%$ are underlined.

shown the same restriction sites for NaeI, HinfI, and FokI. $M$. xenopi, $M$. fortuitum, and $M$. chelonae, on the other hand, have none of these four restriction sites (Fig. 5).

\section{DISCUSSION}

In this report, we determined the nucleotide sequences of the dnaJ gene segments of 19 mycobacterial species obtained by PCR amplification. We compared these nucleotide sequences, deduced their amino acid sequences, and constructed phylogenetic trees of mycobacterial species by the distance matrix methods. We also determined the speciesspecific restriction sites, which were found to be useful for differentiating most pathogenic mycobacteria.

To amplify a common spectrum among the mycobacterial species, we used the $d n a J$ gene encoding one of the stress proteins, dnaJ, as a target. Stress proteins have been universally conserved from bacteria to man, and those sequences are at least $50 \%$ identical to each other at the amino acid level (1). Sequence similarity of dnaJ between Escherichia coli and $M$. tuberculosis is approximately $70 \%(14)$, and dnaJ proteins among the mycobacterial species are considered to be more conserved. Thus, we expected that the dnaJ gene would be suitable as a target for genus-specific PCR and identification of the mycobacterial species based on the nucleotide sequence differences.

In the present study, we found that there were several peculiar nucleotide sequence differences among the 19 amplified mycobacterial dnaJ genes. These differences have provided useful information to differentiate and discriminate the mycobacterial species. The $M$. tuberculosis group (including $M$. bovis, BCG, $M$. africanum, and $M$. microti) is seen to form a genetically distinct cluster from the nontuberculous mycobacteria, because two portions of the nucleotide sequences are clearly different from each other. In particular, one nucleotide substitution (position 1442) accompanied by one amino acid change from glutamine to lysine is a distinctive feature, indicating the difference between the tuberculous and nontuberculous mycobacteria.

To clarify mycobacterial phylogeny and its relationship to mycobacterial taxonomy, we constructed a phylogenetic tree by the neighbor-joining method, which formed a phylogenetic tree with three major branches. To evaluate the confidence limit of the tree constructed by the neighborjoining method in this study, we made use of the bootstrapping technique (7). Where parts of the tree were weakly supported by the sequence data, different topologies (branching orders) are given. As a consequence of these analyses, the division into three groups was considered significant, because $P$ values were less than 0.05 . The first group formed a large branch consisting of the $M$. tuberculosis complex, $M$. avium, $M$. intracellulare, $M$. paratuberculosis, $M$. hemophilum, and $M$. simiae. Although known to be a member of the slowly growing photochromogens (Runyon's group I), $M$. simiae was found to be associated with the cluster including the slowly growing nonphotochromogens (Runyon's group III) in our phylogenetic analysis. The second group consisted of $M$. chelonae, a rapid grower, as well as three slowly growing photochromogens (Runyon's group I), namely, $M$. kansasii, $M$. gastri, and $M$. marinum. The third group was made up of three slowly growing scotochromogens (Runyon's group II), $M$. scrofulaceum, $M$. gordonae, and $M$. szulgai. However, a rapid grower, $M$. fortuitum, was not significantly connected with this group because the confidence limit by bootstrapping was considerably lower. $M$. xenopi formed an independent branch by itself. To confirm the phylogenetic relationship of the mycobacterial species based on the dnaJ sequences, we constructed another phylogenetic tree by UPGMA. The rooted phylogenetic tree was inferred, and its topological relationship was very similar to the tree inferred by the neighborjoining method except for the line consisting of $M$. hemo- 


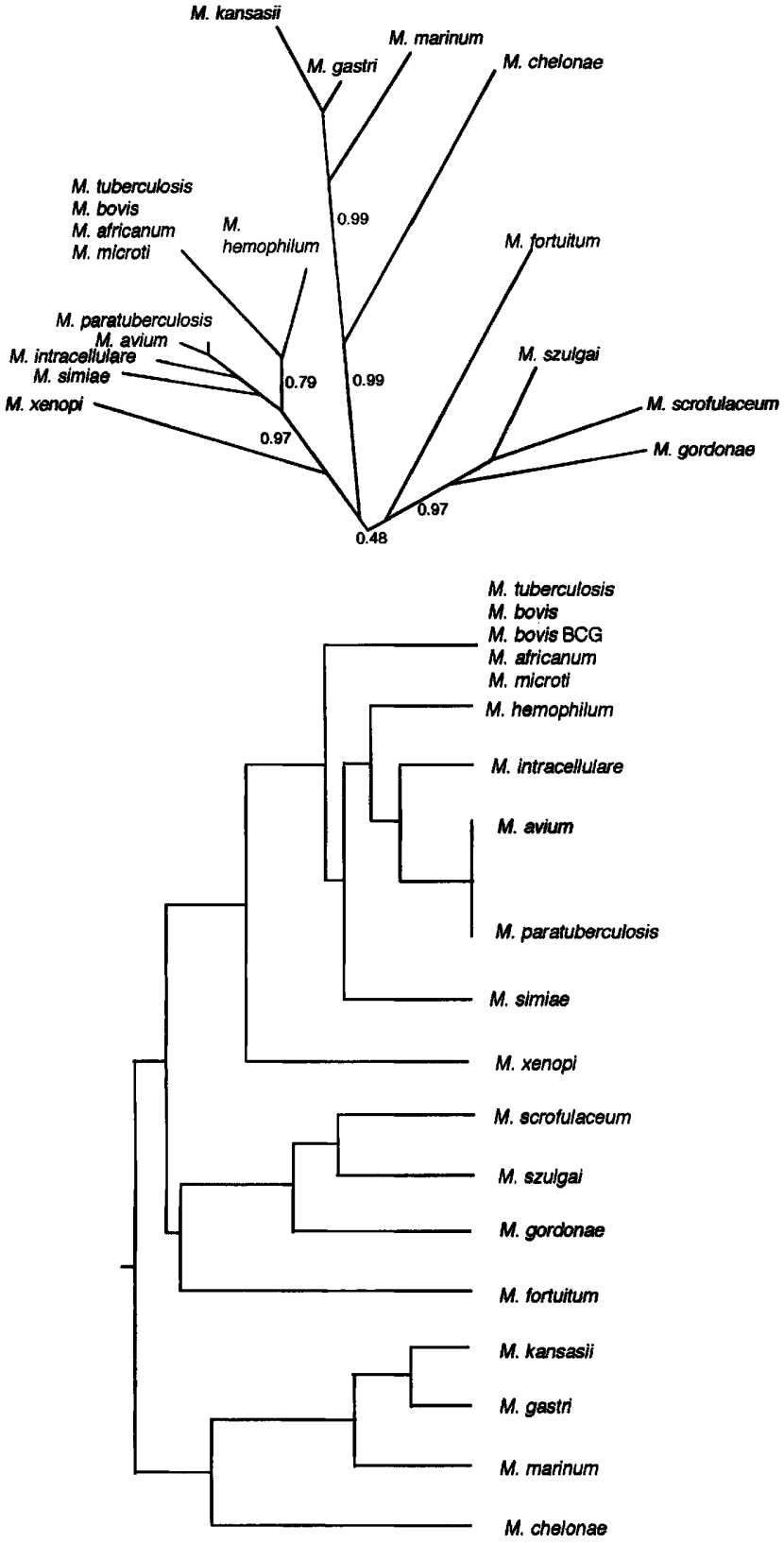

FIG. 3. (Top) Phylogenetic tree of mycobacterial dnaJ genes by the neighbor-joining method. The evolutionary distance between two species is the sum of the branch lengths between them. The values in the phylogenetic tree represent bootstrap probabilities. (Bottom) Phylogenetic tree showing the relationship of the mycobacterial species. The relationship was derived by UPGMA.

philum. Nei pointed out that when the phylogenetic trees inferred by both the neighbor-joining method and UPGMA are the same, the phylogenetic relationship determined by the distance matrix methods seems reliable (17). Therefore, we suggest that mycobacterial phylogeny derived from the nucleotide sequence analysis of the dnaJ gene is relevant.

Recently, three groups reported a phylogenetic relationship between slow and rapid growers of the genus Mycobacterium on the basis of 16S rRNA nucleotide sequence differences $(20,23,34)$. They indicated that slowly growing species of mycobacteria have natural coherence, resulting in

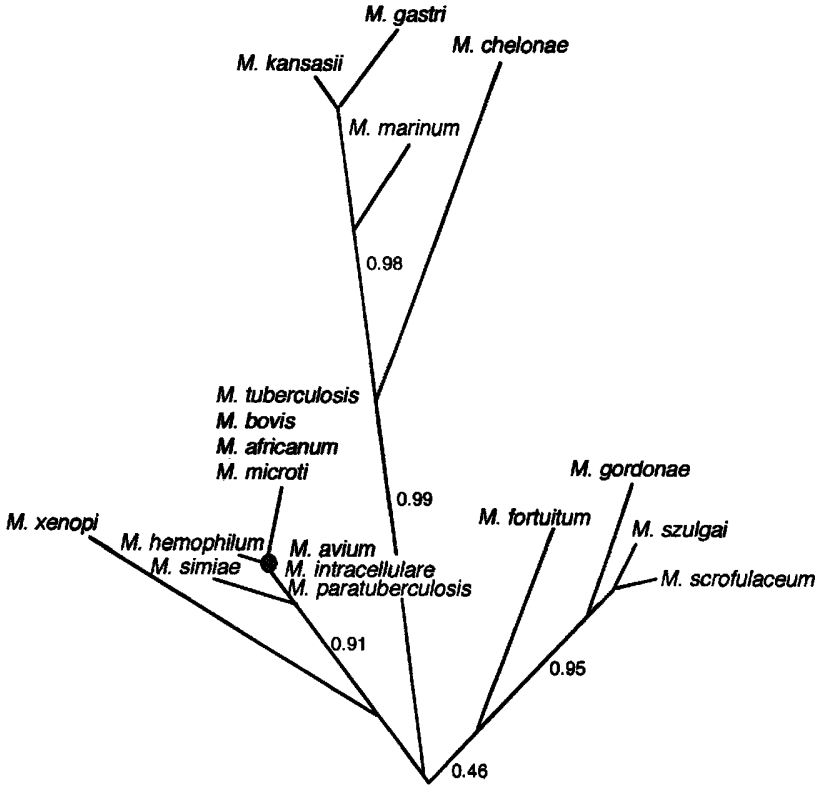

FIG. 4. Phylogenetic relationship inferred from deduced amino acid differences. The values in the tree indicate bootstrap probabilities.

a distinct line of evolutionary descent separable from that of the rapidly growing species. However, distinct clusters were not revealed within the slowly growing line of descent as is found with Runyon's groups. The phylogenetic tree inferred

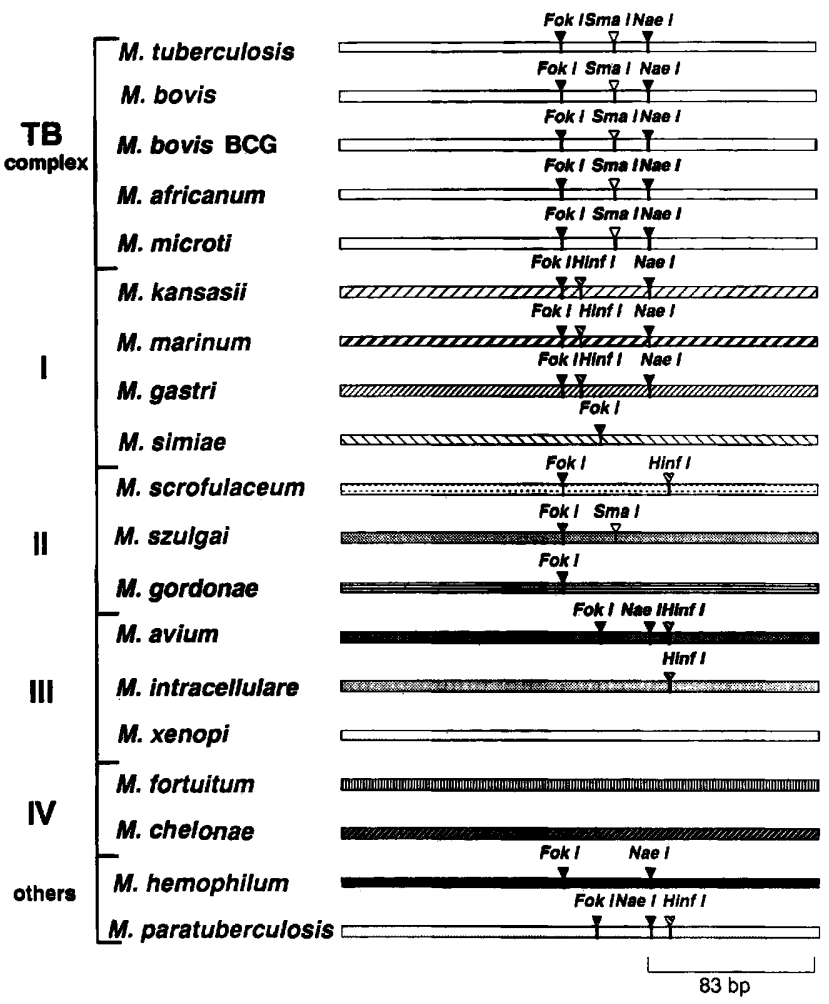

FIG. 5. Restriction map of the dnaJ gene of 19 mycobacterial species between sequences 1394 and 1629. Restriction sites for SmaI, NaeI, HinfI, and FokI are indicated. TB, tuberculosis. Runyon's groups are indicated by Roman numerals. 

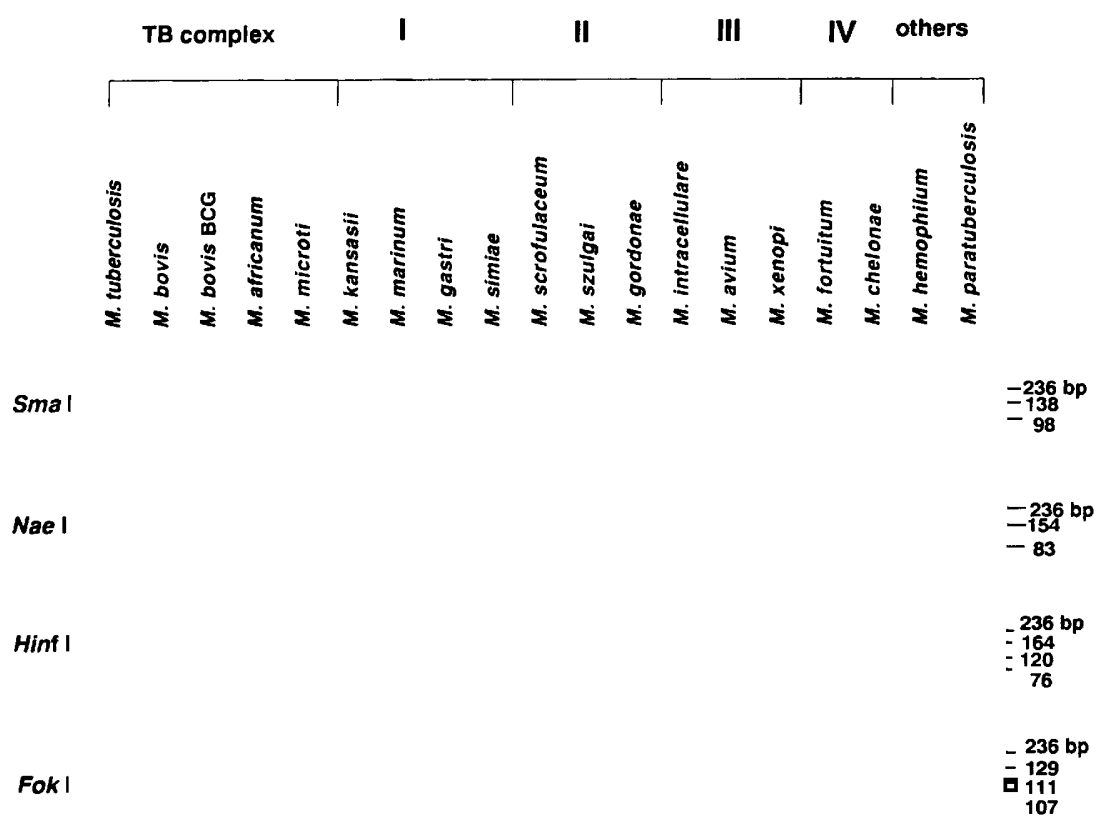

FIG. 6. Endonuclease digestions of the PCR products of the dnaJ gene by SmaI, NaeI, Hinfl, and FokI in the same 19 mycobacterial species described in Fig. 5. Note that most of the mycobacterial species can be differentiated by analyzing the restriction patterns with $S m a I$, NaeI, HinfI, and FokI. TB, tuberculosis. Runyon's groups are indicated by Roman numerals.

based on the dnaJ gene sequences is in better agreement with phenotypic characteristics than that based on the $16 \mathrm{~S}$ rRNA genes as far as slowly growing species are concerned. Because the dnaJ gene is a protein-coding gene, we further constructed the phylogenetic tree inferred from the amino acid sequence differences of dnaJ by the neighbor-joining method. The topologies of the trees derived from the nucleotide and amino acid sequence analyses were very close to one another. From these observations, the phylogenetic relationship based on the $16 \mathrm{~S}$ rRNA gene sequences seems to reflect the growth rate of fast-growing mycobacteria, whereas the phylogenetic tree derived from the $d n a J$ gene sequence analysis seems to reflect the chromogenic characteristics in slowly growing mycobacterial species. Recently, Wasem et al. (39) reported that when the 20 enzyme activities were detected by multilocus enzyme electrophoresis analysis of mycobacteria, the genetic clustering of the mycobacterial species correlated somewhat with the Runyon groupings. Further nucleotide sequence analyses of many other genes should be conducted to investigate the differences in phylogenetic relationship among the mycobacterial species based on protein-coding genes and RNA genes.

Finally, in this study, we also identified the speciesspecific restriction sites within the PCR products of the dnaJ gene and found that the $M$. tuberculosis complex can be rapidly and effectively discriminated from nontuberculous mycobacteria by PCR-RFLP analysis. The restriction mapping of the dnaJ gene products also indicated that 13 species of nontuberculous mycobacteria could be divided into nine groups with four restriction enzymes, SmaI, NaeI, HinfI, and FokI. $M$. avium and $M$. intracellulare, which cannot be differentiated by conventional culture methods, can be readily discriminated by PCR-RFLP analysis. This analysis could not differentiate several mycobacterial species by the four restriction enzyme patterns, but this should be possible when different types of restriction enzymes inferred from the $d n a J$ nucleotide sequences are used. Briefly, $M$. kansasii has an $H a e$ I site at position $1468, M$. marinum has the same enzyme site at position 1575 , and $M$. gastri has no site for this enzyme. $M$. xenopi has a DraIII site at position $1585, M$. fortuitum has an HaeII site at position 1473 , and $M$. chelonae has a SacI site at position 1449. Recently, two groups reported that PCR and subsequent restriction enzyme pattern analysis were useful for rapid identification of mycobacteria at the species level $(22,37)$. Currently, we are also applying our PCR-RFLP analysis to identification of mycobacteria in clinical samples and have already obtained evidence showing that PCR-RFLP is highly reliable for clinical diagnosis of mycobacterial infections.

In summary, nucleotide sequence comparison of the dnaJ gene among mycobacterial species has introduced a new phylogenetic analysis in good agreement with the conventional phenotypic classification within slowly growing species. Furthermore, PCR for the dnaJ gene is useful for detection and identification of mycobacteria at the species level, in particular when genus-specific PCR and subsequent restriction mapping analysis are combined.

\section{ACKNOWLEDGMENTS}

We thank Yoshitaka Goto of the National Institute of Health, Japan, for providing mycobacterial strains.

This study was supported by Japanese Ministry of Education grant-in-aid 02557105.

\section{REFERENCES}

1. Ang, D., K. Liberek, D. Skowyra, M. Zylicz, and C. Georgopoulos. 1991. Biological role and regulation of the universally conserved heat shock proteins. J. Biol. Chem. 266:24233-24236.

2. Baess, I. 1979. Deoxyribonucleic acid relatedness among species of slowly growing mycobacteria. Acta Pathol. Microbiol. Immunol. Scand. Sect. B 87:221-226.

3. Baess, I. 1982. Deoxyribonucleic acid relatedness among species of rapidly growing mycobacteria. Acta Pathol. Microbiol. Immunol. Scand. Sect. B 90:371-375.

4. Böddinghaus, B., T. Rogall, T. Flohr, H. Blöcker, and E. C. 
Böttger. 1990. Detection and identification of mycobacteria by amplification of rRNA. J. Clin. Microbiol. 28:1751-1759.

5. Brisson-Noel, A., B. Gicquel, D. Lecossier, V. Lévy-Frébault, X. Nassif, and A. J. Hance. 1989. Rapid diagnosis of tuberculosis by amplification of mycobacterial DNA in clinical samples. Lancet ii: $1069-1071$

6. Eisenach, K. D., M. D. Cave, J. H. Bates, and J. T. Crawford. 1990. Polymerase chain reaction amplification of a repetitive DNA sequence specific for Mycobacterium tuberculosis. J. Infect. Dis. 161:977-981.

7. Felsenstein, J. 1985. Confidence limits on phylogenies: an approach using the bootstrap. Evolution 39:783-791.

8. Georgopoulos, C., D. Ang, K. Liberek, and M. Zylicz. 1990. Stress proteins in biology and medicine, p. 191-221. Cold Spring Harbor Laboratory, Cold Spring Harbor, New York.

9. Gonzalez, R., and B. A. Hanna. 1987. Evaluation of Gen-Probe DNA hybridization systems for the identification of Mycobacterium tuberculosis and Mycobacterium avium-intracellulare. Diagn. Microbiol. Infect. Dis. 8:69-78.

10. Gross, W. M., and L. G. Wayne. 1970. Nucleic acid homology in the genus Mycobacterium. J. Bacteriol. 104:630-634.

11. Horsburgh, C. R., Jr. 1991. Mycobacterium avium complex infection in the acquired immunodeficiency syndrome. N. Engl. J. Med. 324:1332-1338.

12. Imaeda, T., G. Broslawski, and S. Imaeda. 1988. Genomic relatedness among mycobacterial species by nonisotopic blot hybridization. Int. J. Syst. Bacteriol. 38:151-156.

13. Kwok, S., and R. Higuchi. 1989. Avoiding false positives with PCR. Nature (London) 339:237-238.

14. Lathigra, R. B., D. B. Young, D. Sweeter, and R. A. Young. 1988. A gene from $M$. tuberculosis which is homologous to the $d n a J$ heat shock protein of $E$. coli. Nucleic Acids Res. 16:1636.

15. Minnikin, D. E., S. M. Minnikin, J. H. Parlett, M. Goodfellow, and M. Magnusson. 1984. Mycolic acid patterns of some species of Mycobacterium. Arch. Microbiol. 139:225-231.

16. Musial, C. E., L. S. Tice, L. Stockman, and G. D. Roberts. 1988. Identification of mycobacteria from culture using the Gen-Probe rapid diagnostic system for Mycobacterium avium complex and Mycobacterium tuberculosis complex. J. Clin. Microbiol. 26: 2120-2123.

17. Nei, M. 1987. Molecular evolutionary genetics. Columbia University Press, New York.

18. Ogawa, T., and K. Sada. 1949. The quantitative culture method for tubercle bacilli: on the case of cultivation of bacterial suspension. Kekkaku 24:13-18.

19. Pierre, C., D. Lecossier, Y. Boussougant, D. Bocart, V. Joly, P. Yeni, and A. J. Hance. 1991. Use of a reamplification protocol improves sensitivity of detection of Mycobacterium tuberculosis in clinical samples by amplification of DNA. J. Clin. Microbiol. 29:712-717.

20. Pitulle, C., M. Dorsch, J. Kazda, J. Wolters, and E. Stackebrandt. 1992. Phylogeny of rapidly growing members of the genus Mycobacterium. Int. J. Syst. Bacteriol. 42:337-343.

21. Plikaytis, B. B., R. H. Gelber, and T. M. Shinnick. 1990. Rapid and sensitive detection of Mycobacterium leprae using a nestedprimer gene amplification assay. J. Clin. Microbiol. 28:19131917.

22. Plikaytis, B. B., B. D. Plikaytis, M. A. Yakrus, W. R. Butler, C. L. Woodlay, V. A. Silcox, and T. M. Shinnick. 1992. Differentiation of slowly growing Mycobacterium species, including Mycobacterium tuberculosis, by gene amplification and restriction fragment length polymorphism analysis. J. Clin. Microbiol. 30:1815-1822.

23. Rogall, T., J. Wolters, T. Flohr, and E. Böttger. 1990. Towards a phylogeny and definition of species at the molecular level within the genus Mycobacterium. Int. J. Syst. Bacteriol. 40: 323-330.

24. Runyon, E. H. 1959. Anonymous mycobacteria in pulmonary disease. Med. Clin. North Am. 43:273-290.

25. Runyon, E. H. 1970 . Identification of mycobacterial pathogens utilizing colony characteristics. Am. J. Clin. Pathol. 54:578-586.

26. Saiki, R. K., S. Scharf, F. Faloona, K. B. Mullis, G. T. Horn, H. A. Erlich, and N. Arnheim. 1985. Enzymatic amplification of $\beta$-globin genomic sequences and restriction site analysis for diagnosis of sickle cell anemia. Science 230:1350-1354.

27. Saitou, N., and M. Nei. 1987. The neighbor-joining method: a new method for reconstructing phylogenetic trees. Mol. Biol. Evol. 4:406-425.

28. Sambrook, J., E. F. Fritsch, and T. Maniatis. 1989. Molecular cloning: a laboratory manual, 2nd ed. Cold Spring Harbor Laboratory, Cold Spring Harbor, N.Y.

29. Sanger, F., S. Nicklen, and A. R. Coulson. 1977. DNA sequencing with chain-terminating inhibitors. Proc. Natl. Acad. Sci. USA 74:5463-5467.

30. Shanker, P., N. Manjunath, K. K. Mohan, K. Prasad, M. Behari, Shriniwas, and G. K. Ahuja. 1991. Rapid diagnosis of tuberculosis meningitis by polymerase chain reaction. Lancet 337:5-7.

31. Shapiro, H. S., G. A. Splitter, and R. A. Welch. 1988. Deoxyribonucleic acid relatedness of Mycobacterium paratuberculosis to other members of the family Mycobacteriaceae. Int. J. Syst. Bacteriol. 38:143-146.

32. Sjöbring, U., M. Mecklenburg, A. B. Andersen, and H. Miörner. 1990. Polymerase chain reaction for detection of Mycobacterium tuberculosis. J. Clin. Microbiol. 28:2200-2204.

33. Sommers, H. M., and R. C. Good. 1985. Mycobacterium, p. 216-248. In E. H. Lennette, A. Balows, W. J. Hausler, Jr., H. J. Shadomy (ed.), Manual of clinical microbiology, 4th ed. American Society for Microbiology, Washington, D.C.

34. Stahl, D. A., and J. W. Urbance. 1990. The division between fast- and slow-growing species corresponds to natural relationships among the mycobacteria. J. Bacteriol. 172:116-124.

35. Takewaki, S., K. Okuzumi, H. Ishiko, K. Nakahara, A. Ohkubo, and R. Nagai. 1993. Genus-specific polymerase chain reaction for the mycobacterial $d n a J$ gene and species-specific oligonucleotide probes. J. Clin. Microbiol. 31:446-450.

36. Tanimura, M., K. Miyamura, and N. Takeda. 1985. Construction of a phylogenetic tree of enterovirus 70 . Jpn. J. Genet. 60:137-150.

37. Telenti, A., F. Marchesi, M. Balz, F. Bally, E. C. Böttger, and T. Bodmer. 1993. Rapid identification of mycobacteria to the species level by polymerase chain reaction and restriction enzyme analysis. J. Clin. Microbiol. 31:175-178.

38. Tuboly, S. 1965. Studies on the antigenic structure of mycobacteria. I. Comparison of the antigenic structure of pathogenic structure and saprophytic mycobacteria. Acta Microbiol. Acad. Sci. Hung. 12:233-240.

39. Wasem, C. F., C. M. McCarthy, and L. W. Murray. 1991. Multilocus enzyme electrophoresis analysis of the Mycobacterium avium complex and other mycobacteria. J. Clin. Microbiol. 29:264-271.

40. Wit, D. D., L. Steyn, S. Shoemaker, and M. Sogin. 1990. Direct detection of Mycobacterium tuberculosis in clinical specimens by DNA amplification. J. Clin. Microbiol. 28:2437-2441.

41. Young, D., R. Lathigra, R. Hendrix, D. Sweeter, and R. A. Young. 1988. Stress proteins are immune targets in leprosy and tuberculosis. Proc. Natl. Acad. Sci. USA 85:4267-4270. 